\title{
On topological soft sets
}

\author{
M. BURÇ KANDEMIR AND B. TANAY
}

\begin{abstract}
In this paper, we have established topological soft sets over generalized topological spaces and topological spaces, and studied its structural properties. We have derived a topological soft set in any given topological space, and from this point of view, we have given necessary and sufficient condition for homeomorphic Alexandroff spaces using topological soft set technique. At last, we have derived a topological soft set using closed sets in any topological space and we have given necessary and sufficient condition for arbitrary homeomorphic topological spaces using them.
\end{abstract}

Mathematics Subject Classification 2010: AMS 54A05, 54C10, 54C50, 54C60.

Keywords: Soft set, topology, generalized topology, soft topology, topological soft set.

\section{INTRODUCTION}

Topology is a major area of mathematics concerned with the most properties of space. Topological spaces show up naturally in almost every branch of mathematics and other sciences. This makes topology one of the great unifying ideas of mathematics. In 2002, Császár defined the concept of generalized topology which is the family closed under arbitrary union of subsets of a set in [6]. At the same time, many scientists use the topology or the generalized topology to understand and model the real world. But this is not always easy. Each phenomenon in the real world can not always be modeled by classical methods. Many theories have been developed for dealing with uncertainties. One of them is the soft set theory which has a rich potential for applications in several directions. The notion of soft set theory was initiated by Molodtsov [16] in 1999. As in [2, 18], this theory have been applied to many area in mathematics, information science and computer science. Of course, most of the mathematicians studied the topological structure on soft sets. Firstly, Shabir and Naz established the concept of soft topological space which is defined over an initial universe with a fixed set of parameters in [20]. They introduced soft open sets, soft closed sets, soft closure, soft interior points, soft neighborhood of a point and soft separation axioms. In [8], Ge et al. gave some relations between topology and soft set theory. They presented some characterizations of trivial 
(discrete, $T_{0}, T_{1}, T_{D}$, and $R_{0}$ ) topological spaces by the null soft set, the absolute soft set, the identical soft set and so on. In [11], Li et al. prepared new characterization between topology and soft set theory. They defined the concept of topological soft set which is a soft set over initial universe such that for each parameter this is topology over initial universe, and studied relations between approximation spaces. But this definition of topological soft set is very specific and restricted. If we have a topological space or more general generalized topological space, then we can get a more convenient way types of topological soft sets. For this reason, in this paper, we re-define the concept of topological soft set in any generalized topological space without any restriction and study its properties.

\section{PRELIMINARIES}

As the preliminary information, which is necessary to study, give some definitions and properties.

\subsection{Soft Set Theory}

Let $U$ be an initial universe, $E$ be a set of parameters, $\mathscr{P}(U)$ be the power set of $U$, and $A \subseteq E$. Molodtsov [16] defined the soft set in the following manner:

DEFinition 2.1. [16] A pair $(F, A)$ is called a soft set over $U$ where $F$ is a mapping given by $F: A \rightarrow \mathscr{P}(U)$.

Some set-theoretic operations defined by $[12,18]$

Definition 2.2. [18] For two soft sets $(F, A)$ and $(G, B)$ over a common universe $U$, we say that $(F, A)$ is a soft subset of $(G, B)$ and is denoted by $(F, A) \widetilde{\subset}(G, B)$ if

(i) $A \subset B$ and,

(ii) $\forall a \in A, F(a) \subset G(a)$.

Definition 2.3. [18] Two soft sets $(F, A)$ and $(G, B)$ over a common universe $U$ are said soft equal if $(F, A)$ is a soft subset of $(G, B)$, and $(G, B)$ is a soft subset of $(F, A)$.

Definition 2.4. [18] Let $(F, A)$ and $(G, B)$ be two soft sets over a common universe $U$ such that $A \cap B \neq \varnothing$. The intersection of $(F, A)$ and $(G, B)$ is denoted by 
$(F, A) \widetilde{\cap}(G, B)$, and is defined as $(F, A) \widetilde{\cap}(G, B)=(H, C)$, where $C=A \cap B$ and for all $c \in C, H(c)=F(c) \cap G(c)$.

Definition 2.5. [12] The union of two soft sets $(F, A)$ and $(G, B)$ over a common universe $U$ is the soft set $(H, C)$, denoted by $(F, A) \widetilde{\cup}(G, B)=(H, C)$, where $C=A \cup B$, and $\forall c \in C$,

$$
H(c)= \begin{cases}F(c) & , \text { if } c \in A-B \\ G(c) & , \text { if } c \in B-A \\ F(c) \cup G(c) & , \text { if } c \in A \cap B\end{cases}
$$

DEFINITION 2.6. [4] Let $U$ be an initial universe set, $E$ be the universe set of parameters, and $A \subset E$.

(i) $(F, A)$ is called a relative null soft set (with respect to the parameter set $A$ ), $\operatorname{denoted}$ by $\Phi_{A}$, if $F(a)=\varnothing$ for all $a \in A$.

(ii) $(F, A)$ called a relative whole soft set (with respect to the parameter set $A$ ), $\operatorname{denoted}$ by $\mathscr{U}_{A}$, if $F(a)=U$ for all $a \in A$.

The relative whole soft set $\mathscr{U}_{E}$ with respect to the universe set of parameters $E$ is called the absolute soft set over $U$.

DeFinition 2.7. [12] Let $(F, A)$ and $(G, B)$ be two soft sets over the common universe $U$. Then $(F, A)$ AND $(G, B)$ denoted by $(F, A) \wedge(G, B)$ and is defined by $(F, A) \wedge$ $(G, B)=(H, A \times B)$ where $H((a, b))=F(a) \cap G(b)$, for all $(a, b) \in A \times B$.

DeFinition 2.8. [12] Let $(F, A)$ and $(G, B)$ be two soft sets over the common universe $U$. Then $(F, A)$ OR $(G, B)$ denoted by $(F, A) \vee(G, B)$ and is defined by $(F, A) \vee$ $(G, B)=(H, A \times B)$ where $H((a, b))=F(a) \cup G(b)$, for all $(a, b) \in A \times B$.

DEFINITION 2.9. [18] The complement of a soft set $(F, A)$ is denoted by $(F, A)^{c}$ and is defined by $(F, A)^{c}=\left(F^{c}, A\right)$, where $F^{c}: A \rightarrow \mathscr{P}(U)$ is a mapping given by $F^{c}(a)=$ $U-F(a)$ for all $a \in A$.

In [14], Min has introduced the concept of similarity between soft sets and investigated some properties. He defined the concept of similarity between soft sets as follows:

DEFINITION 2.10. [14] Let $(F, A)$ and $(G, B)$ be soft sets over a common universe set $U$. Then $(F, A)$ is similar to $(G, B)$ (simply $(F, A) \cong(G, B))$ if there exists a bijection function $\phi: A \rightarrow B$ such that $F(x)=(G \circ \phi)(x)$ for every $x \in A$, where $(G \circ \phi)(x)=$ $G(\phi(x))$. 


\subsection{Soft Mappings}

Kharal and Ahmad [9], defined the notion of a mapping on soft classes and studied several properties of images and inverse images of soft sets supported by examples and counterexamples. They defined that image and inverse image of a soft set under a soft functions as follows:

Definition 2.11. [9] Let $U_{1}, U_{2}$ be initial universes, $E_{1}, E_{2}$ be parameters sets, $\varphi$ be a function from $U_{1}$ to $U_{2}$ and $\psi$ be a function from $E_{1}$ to $E_{2}$. Then the pair $(\varphi, \psi)$ is called soft function from $S\left(U_{1}, E_{1}\right)$ to $S\left(U_{2}, E_{2}\right)$. The image of each $(F, A) \in S\left(U_{1}, E_{1}\right)$ under the soft function $(\varphi, \psi)$ is denoted by $(\varphi, \psi)(F, A)=(\varphi F, \psi(A))$ and is defined as following;

$$
(\varphi F)(\beta)= \begin{cases}\varphi\left(\bigcup_{\alpha \in \psi^{-1}(\beta) \cap A} F(\alpha)\right) & , \psi^{-1}(\beta) \cap A \neq \varnothing \\ \varnothing & , \text { otherwise }\end{cases}
$$

for each $\beta \in \psi(A)$.

Similarly, the inverse image of each $(G, B) \in S\left(U_{2}, E_{2}\right)$ under the soft function $(\varphi, \psi)$ is denoted by $(\varphi, \psi)^{-1}(G, B)=\left(\varphi^{-1} G, \psi^{-1}(B)\right)$ and is defined as following;

$$
\left(\varphi^{-1} G\right)(\alpha)= \begin{cases}\varphi^{-1}(G(\psi(\alpha))) & , \psi(\alpha) \in B \\ \varnothing & , \text { otherwise }\end{cases}
$$

for each $\alpha \in \psi^{-1}(B)$.

\subsection{Soft Equality Relations}

Qin and Hong introduced the concept of soft equality relations $\approx_{S}$ and $\approx^{S}$ in [19]. Definition of soft equalities are given as follows;

Definition 2.12. [19] Let $(F, A),(G, B)$ be two soft sets over $U$.

$\left(\approx_{S}\right) .(F, A)$ is called soft equal to $(G, B)$, denoted by $(F, A) \approx_{S}(G, B)$, if for all $e \in A \cup B$, $e \in A \cap B$ implies $F(e)=G(e), e \in A-B$ implies $F(e)=\varnothing$, and $e \in B-A$ implies $G(e)=\varnothing$.

$\left(\approx^{S}\right) .(F, A)$ is called soft equal to $(G, B)$, denoted by $(F, A) \approx^{S}(G, B)$, if for all $e \in A \cup B$, $e \in A \cap B$ implies $F(e)=G(e), e \in A-B$ implies $F(e)=U$, and $e \in B-A$ implies $G(e)=U$.

Note that these relations are equivalence relations on the family of all soft sets over $U$. 


\section{TOPOLOGICAL SOFT SETS}

In topology, topologists defined some generalization of open sets such as semi-openness [10], pre-openness [13], $\alpha$-openness [17], $\beta$-openness [1] etc. In [5] and [6], Császár gave a frame of generalized openness as follows;

Let $U$ be a set and $\gamma$ be a map from $\mathscr{P}(U)$ into itself. Suppose that $\gamma$ is monotonic, i.e. $A \subseteq B \subseteq U$ implies $\gamma(A) \subseteq \gamma(B)$. A set $A \subseteq U$ is called $\gamma$-open if and only if $A \subseteq \gamma(A)$, and $B \subseteq U$ is called $\gamma$-closed if its complement is $\gamma$-open. He showed that any union of $\gamma$-open sets is $\gamma$-open in [5]. So, he defined the notion of generalized topology which is constituted by $\gamma$-open sets in [6]. The collection $\mathfrak{g}$ of subsets of $U$ is called generalized topology on $U$ if and only if $\varnothing \in \mathfrak{g}$ and $G_{i} \in \mathfrak{g}$ for $i \in I \neq \varnothing$ implies $G=\bigcup_{i \in I} G_{i} \in \mathfrak{g}$. If $\mathfrak{o}$ is a topology on $U$ in the usual sense and denote by $i A$ the $\mathfrak{o}$-interior int $A$, by $c A$ the $\mathfrak{o}$-closure $c l A$, we obtain as important particular cases the collection $\mathfrak{o}$ of all open sets $(\gamma=i), \mathfrak{s o}$ of all semi-open sets $(\gamma=c i)$, po of all preopen sets $(\gamma=i c), \beta \mathfrak{o}$ of all $\beta$-open sets $(\gamma=c i c), \alpha \mathfrak{o}$ of all $\alpha$-open sets $(\gamma=i c i)[6]$. Therefore, we obtain the relationship

$$
\mathfrak{o} \subset \alpha \mathfrak{o} \subset \mathfrak{s o} \subset \beta \mathfrak{o} \subset \mathfrak{g}
$$

and

$$
\mathfrak{o} \subset \alpha \mathfrak{o} \subset \mathfrak{p o} \subset \mathfrak{g}
$$

from [5-7]. In a similar manner, we obtain that $K$ is closed iff $c(K) \subset K, K$ is semiclosed iff $i c(K) \subset K, K$ is pre-closed iff $\operatorname{ci}(K) \subset K, K$ is $\alpha$-closed iff $\operatorname{cic}(K) \subset K$ and $K$ is $\beta$-closed iff $i c i(K) \subset K$. Then we denote the families of all type closed sets as $\mathfrak{c}, \mathfrak{s c}$, $\mathfrak{p c}, \alpha \mathfrak{c}$ and $\beta \mathfrak{c}$, respectively. We also denote the family of generalized closed set in the sense of Császár by $\mathfrak{g c}$.

We noted that $\mathfrak{o}$ and $\alpha \mathfrak{o}$ are topologies on $U$ and we obtain that $\mathfrak{o}$ is coarser than $\alpha \mathfrak{o}$ from the above relationships.

In [6], Császár define the concept of generalized continuity of any function from a generalized topological space to another. So, given two generalized topological spaces $\left(U_{1}, \mathfrak{g}_{1}\right)$ and $\left(U_{2}, \mathfrak{g}_{2}\right)$ and a mapping $f: U_{1} \rightarrow U_{2}, f$ is $\left(\mathfrak{g}_{1}, \mathfrak{g}_{2}\right)$-continuous iff $G \in \mathfrak{g}_{2}$ implies that $f^{-1}(G) \in \mathfrak{g}_{1}$ [6]. We shortly say that $f$ is $\mathfrak{g}$-continuous if $f$ is generalized continuous. We call that $f$ is $\mathfrak{g}$-open ( $\mathfrak{g}$-closed, respectively) if $G \in \mathfrak{g}$ implies that $f(G) \in \mathfrak{g}^{\prime}$ (or $G \in \mathfrak{g} \mathfrak{c} \Rightarrow f(G) \in \mathfrak{g}^{\prime} \mathfrak{c}$ ). We also call that $f$ is $\mathfrak{g}$-homeomorphism if $f$ is 
bijective, $\mathfrak{g}$-continuous and $\mathfrak{g}$-open (or $\mathfrak{g}$-closed).

Now, we can define $\gamma$-topological soft set over an initial universe space as the follows;

DEFINITION 3.1. Let $U$ be an initial universe and $\gamma$ be a monotonic map from $\mathscr{P}(U)$ into itself. $(F, A)$ be a soft set over $U$ where $A \subseteq E .(F, A)$ is called $\gamma$-topological soft set if $F(e)$ is $\gamma$-open set in $U$ i.e. $F(e) \subseteq \gamma(F(e))$ for all $e \in A$.

In Definition 3.1, in the most general sense, we can conclude that there is a generalized topology on $U$ because of that obtained by $\gamma$-open sets. Therefore, we can explain this definition as " $(F, A)$ is a $\mathfrak{g}$-topological soft set over $U$ if and only if $F(e) \in \mathfrak{g}$ for all $e \in A$ where $\mathfrak{g}$ is a generalized topology on $U$ ". If we take the $\gamma$ as interior operator, then we get the classical topology on $U$. In [15], W. K. Min defined that $(F, A)$ is open soft set over the topological space $U$ such that $F(e)$ is open for all $e \in A$ i.e. $F(e) \subseteq i(F(e))$ for all $e \in A$. From his definition, we obtain that $(F, A)$ is an o-topological soft set over $U$ in our sense. Clearly, every open soft set is an o-topological soft set. Furthermore, we can characterize given any $\gamma$-topological soft set according to type of openness. For this, we can give following definition:

DEFINITION 3.2. Let $U$ be an initial universe, $E$ be a parameters set, $A \subseteq E$ and $(F, A)$ be a soft set over $U$.

(1) $(F, A)$ is called $\mathfrak{g}$-topological soft set if $F(e) \in \mathfrak{g}$ for all $e \in A$.

(2) $(F, A)$ is called o-topological soft set if $F(e) \in \mathfrak{o}$ for all $e \in A$.

(3) $(F, A)$ is called $\mathfrak{s o - t o p o l o g i c a l ~ s o f t ~ s e t ~ i f ~} F(e) \in \mathfrak{s o}$ for all $e \in A$.

(4) $(F, A)$ is called po-topological soft set if $F(e) \in \mathfrak{p o}$ for all $e \in A$.

(5) $(F, A)$ is called $\alpha \mathfrak{o}$-topological soft set if $F(e) \in \alpha \mathfrak{o}$ for all $e \in A$.

(6) $(F, A)$ is called $\beta$ o-topological soft set if $F(e) \in \beta \mathfrak{o}$ for all $e \in A$.

We also can characterize the topological soft sets according to type of closedness as follows.

Definition 3.3. Let $U$ be an initial universe, $E$ be a parameters set, $A \subseteq E$ and $(F, A)$ be a soft set over $U$.

(1) $(F, A)$ is called $\mathfrak{g c}$-topological soft set if $F(e) \in \mathfrak{g c}$ for all $e \in A$.

(2) $(F, A)$ is called $\mathfrak{c}$-topological soft set if $F(e) \in \mathfrak{c}$ for all $e \in A$.

(3) $(F, A)$ is called $\mathfrak{s c}$-topological soft set if $F(e) \in \mathfrak{s c}$ for all $e \in A$. 
(4) $(F, A)$ is called $\mathfrak{p c}$-topological soft set if $F(e) \in \mathfrak{p c}$ for all $e \in A$.

(5) $(F, A)$ is called $\alpha \mathfrak{c}$-topological soft set if $F(e) \in \alpha \mathfrak{c}$ for all $e \in A$.

(6) $(F, A)$ is called $\beta \mathfrak{c}$-topological soft set if $F(e) \in \beta \mathfrak{c}$ for all $e \in A$.

EXAMPLE 3.4. Let $\mathbb{R}$ be the set of real numbers with the usual topology and $\mathbb{N}$ be the parameters set. Define the mappings $F: \mathbb{N} \rightarrow \mathscr{P}(\mathbb{R})$ such that $F(n)=(n-1, n+1)$ and $G: \mathbb{N} \rightarrow \mathscr{P}(\mathbb{R})$ such that $G(n)=(n-1, n+1]$. Then $(F, \mathbb{N})$ is an o-topological soft set and $(G, \mathbb{N})$ is a $\mathfrak{s o - t o p o l o g i c a l ~ s o f t ~ s e t s ~ o v e r ~} \mathbb{R}$, respectively. Moreover, if we define the mapping $H: \mathbb{N} \rightarrow \mathscr{P}(\mathbb{R})$ such that $H(n)=[n-1, n+1]$, then we obtain that $(H, \mathbb{N})$ is a $\mathfrak{c}$-topological soft set over $\mathbb{R}$.

EXAMPLE 3.5. Let $U=\{a, b, c\}$ and $\mathfrak{o}=\{\varnothing, U,\{a, b\},\{b, c\},\{b\}\}$ be a topology on $U$. Let $E=\{1,2,3,4,5\}$ and $(F, A)=\{1=\{b\}, 2=\{b, c\}\}$ be a soft set over $U$ with $A \subseteq E$. Then $(F, A)$ is a o-topological soft set over $U$.

Now, we discuss the obtained results.

Firstly, soft subset of a $\gamma$-topological soft set may not be $\gamma$-topological in general. If we specifically give the following example for $\mathfrak{o}$-topological soft sets, we can generalize other type.

EXAMPLE 3.6. From Example 3.5, we have the o-topological soft set $(F, A)$. Although, $(G, B)=\{2=\{c\}\}$ is a soft subset of $(F, A)$, it is not o-topological.

THEOREM 3.7. Let $(F, A)$ be a soft set over $U$, where $A \subseteq E$. Then we have

$$
\begin{aligned}
(F, A) \text { is o-topological } & \Rightarrow(F, A) \text { is } \alpha \mathfrak{o} \text {-topological } \\
& \Rightarrow(F, A) \text { is } \mathfrak{s o} \text {-topological } \\
& \Rightarrow(F, A) \text { is } \beta \mathfrak{o} \text {-topological } \\
& \Rightarrow(F, A) \text { is } \mathfrak{g} \text {-topological }
\end{aligned}
$$

and

$$
\begin{aligned}
(F, A) \text { is o-topological } & \Rightarrow(F, A) \text { is } \alpha \mathfrak{o} \text {-topological } \\
& \Rightarrow(F, A) \text { is } \mathfrak{p o} \text {-topological } \\
& \Rightarrow(F, A) \text { is } \mathfrak{g} \text {-topological }
\end{aligned}
$$

Proof. From Equation (1) and Equation (2), it is obvious. 
THEOREM 3.8. If $(F, A)$ is a $\mathfrak{g}$-topological soft set, then its complement $(F, A)^{c}$ is a $\mathfrak{g c}$-topological soft set.

Proof. Obvious.

THEOREM 3.9. If $(F, A)$ and $(G, B)$ are o-topological (or $\alpha \mathfrak{o}$-topological), then $(F, A) \widetilde{\cap}(G, B)$ is also o-topological (or $\alpha \mathfrak{o}$-topological).

Proof. From Definition 2.4, we obtain the soft set $(H, C)=(F, A) \widetilde{\cap}(G, B)$, such that $C=A \cap B$ and $H(e)=F(e) \cap G(e)$ for all $e \in C$. Since $(F, A)$ and $(G, B)$ are topological, $F(e)$ and $G(e)$ in $\mathfrak{o}$ (or $\alpha \mathfrak{o}$ ) for all $e \in C$, respectively. So, $H(e) \in \mathfrak{o}$ $(H(e) \in \alpha \mathfrak{o})$ for all $e \in A \cap B$. Thus $(H, C)$ is an $\mathfrak{o}$-topological soft set (or $\alpha \mathfrak{o}$-topological soft set) over $U$.

THEOREM 3.10. If $(F, A)$ and $(G, B)$ are $\mathfrak{g}$-topological, then $(F, A) \widetilde{\cup}(G, B)$ is also $\mathfrak{g}$-topological.

Proof. Suppose that, $(F, A) \widetilde{\cup}(G, B)=(H, C)$. Since $(F, A)$ and $(G, B)$ are $\mathfrak{g}$ topological soft sets, we have $H(c)=F(c) \in \mathfrak{g}$ for each $c \in A-B, H(c)=G(c) \in \mathfrak{g}$ for each $c \in B-A$ and $H(c)=F(c) \cup G(c) \in \mathfrak{g}$ for each $c \in A \cap B$ from Definition 2.5. Thus, $(H, C)$ is a $\mathfrak{g}$-topological soft set.

THEOREM 3.11. Null and absolute soft sets are $\mathfrak{g}$-topological.

ProOF. From Definition 2.6 and Definition 3.2, it is obvious.

THEOREM 3.12. If $(F, A)$ and $(G, B)$ are $\mathfrak{o}$-topological (or $\alpha \mathfrak{o}$-topological), then $(F, A) \wedge(G, B)$ is also o-topological (or $\alpha \mathfrak{o}$-topological).

Proof. From Definition 2.7, we have the soft set $(H, A \times B)=(F, A) \wedge(G, B)$, such that $C=A \times B$ and $H(a, b)=F(a) \cap G(b)$ for all $(a, b) \in A \times B$. Since $(F, A)$ and $(G, B)$ are $\mathfrak{o}$-topological (or $\alpha \mathfrak{o}$-topological), then $(H, A \times B)$ is also o-topological (or $\alpha \mathfrak{o}$-topological).

THEOREM 3.13. If $(F, A)$ and $(G, B)$ are $\mathfrak{g}$-topological, then $(F, A) \vee(G, B)$ is also $\mathfrak{g}$-topological.

Proof. From Definition 2.8 and Definition 3.2, the proof of this theorem is similar to the proof of above theorem.

THEOREM 3.14. If $(F, A)$ and $(G, B)$ are $\mathfrak{g c}$-topological, then $(F, A) \widetilde{\cap}(G, B)$ is also $\mathfrak{g c}$-topological.

Proof. Suppose that $(F, A)$ and $(G, B)$ are $\mathfrak{g c}$-topological soft set over $U$ and 
$(F, A) \widetilde{\cap}(G, B)=(H, C)$. Then $C=A \cap B$ and for all $c \in C, H(c)=F(c) \cap G(c)$. Since $(F, A)$ and $(G, B)$ are $\mathfrak{g c}$-topological, $F(a), G(b) \in \mathfrak{g c}$ for all $a \in A$ and $b \in B$. So we obtain that $(F(a))^{c},(G(b))^{c} \in \mathfrak{g}$ for all $a \in A$ and $b \in B$. Since $\mathfrak{g}$ is a generalized topology on $U$. For all $c \in C,(F(c))^{c} \cup(G(c))^{c} \in \mathfrak{g}$. Then, from the rules of de Morgan, we obtain that $[F(c) \cap G(c)]^{c} \in \mathfrak{g}$. Hence $F(c) \cap G(c) \in \mathfrak{g c}$ for all $c \in C$. Thus $(H, C)$ is a $\mathfrak{g c}$-topological soft set over $U$.

THEOREM 3.15. If $(F, A)$ similar to $(G, B)$ and $(F, A)$ is a $\mathfrak{g}$-topological soft set, then $(G, B)$ is also $\mathfrak{g}$-topological.

PROOF. By the definition of similarity (Definition 2.10), we have a bijection $\phi: A \rightarrow B$ such that $F(a)=(G \circ \phi)(a)$ for all $a \in A$. Now, we need to see $G(b) \in \mathfrak{g}$ for all $b \in B$. Since $\phi$ is a bijection, then for all $b \in B$, there exist $a \in A$ such that $\phi(a)=b$. So, for all $b \in B$, we obtain

$$
G(b)=G(\phi(a))=(G \circ \phi)(a)=F(a) .
$$

Since $F(a) \in \mathfrak{g}$ for all $a \in A$, then $G(b) \in \mathfrak{g}$ for all $b \in B$. Thus $(G, B)$ is a topological soft set over $U$.

THEOREM 3.16. Let $(F, A)$ and $(G, B)$ be two soft sets over $(U, \tau)$.

(a) If $(F, A) \approx_{S}(G, B)$ and $(F, A)$ is $\mathfrak{g}$-topological, then $(G, B)$ is also $\mathfrak{g}$-topological.

(b) If $(F, A) \approx^{S}(G, B)$ and $(F, A)$ is $\mathfrak{g}$-topological, then $(G, B)$ is also $\mathfrak{g}$-topological.

Proof. Proof of (a) and (b) is obvious from Definition 2.12 and Definition 3.2.

THEOREM 3.17. Let $\left(U_{1}, \mathfrak{g}_{1}\right)$ and $\left(U_{2}, \mathfrak{g}_{2}\right)$ be generalized topological spaces, $\varphi$ : $U_{1} \rightarrow U_{2}$ and $\psi: E_{1} \rightarrow E_{2}$ be functions and $(F, A)$ be a soft set over $U_{1}$. If $\varphi$ is a $\mathfrak{g}-$ open function and $(F, A)$ is a $\mathfrak{g}$-topological soft set, then its image $(\varphi, \psi)(F, A)$ is a $\mathfrak{g}$-topological soft set over $U_{2}$.

Proof. Since $\varnothing \in \mathfrak{g}_{2}$. If $(\varphi F)(\beta)=\varnothing$ for each $\beta \in \psi(A)$, then $(\varphi F, \psi(A))$ is $\mathfrak{g}$-topological.

Suppose that $(\varphi F)(\beta) \neq \varnothing$. So, $(\varphi F)(\beta)=\varphi\left(\bigcup_{\alpha \in \psi^{-1}(\beta) \cap A} F(\alpha)\right)$ for each $\beta \in \psi(A)$. Since $(F, A)$ is $\mathfrak{g}$-topological and $\varphi$ is a $\mathfrak{g}$-open function, then $\bigcup_{\alpha \in \psi^{-1}(\beta) \cap A} F(\alpha) \in \mathfrak{g}_{2}$. Thus $(\varphi, \psi)(F, A)$ is a $\mathfrak{g}$-topological soft set over $U_{2}$.

THEOREM 3.18. Let $\left(U_{1}, \mathfrak{g}_{1}\right)$ and $\left(U_{2}, \mathfrak{g}_{2}\right)$ be generalized topological spaces, $\varphi$ : $U_{1} \rightarrow U_{2}$ and $\psi: E_{1} \rightarrow E_{2}$ be functions and $(G, B)$ be a soft set over $U_{2}$. If $\varphi$ is a $\mathfrak{g}$-continuous function and $(G, B)$ is a $\mathfrak{g}$-topological soft set, then its inverse image 
$(\varphi, \psi)^{-1}(G, B)$ is a $\mathfrak{g}$-topological soft set over $U_{1}$.

Proof. $\quad$ Since $\varnothing \in \mathfrak{g}_{1}$. If $\left(\varphi^{-1} G\right)(\alpha)=\varnothing$ for each $\alpha \in \psi^{-1}(B)$, then $\left(\varphi^{-1} G, \psi^{-1}(B)\right)$ is $\mathfrak{g}$-topological. Otherwise, $\left(\varphi^{-1} G\right)(\alpha)=\varphi^{-1}(G(\psi(\alpha)))$ for each $\alpha \in \psi^{-1}(B)$. Since $(G, B)$ is $\mathfrak{g}$-topological and $\varphi$ is $\mathfrak{g}$-continuous, we obtain that $(\varphi, \psi)^{-1}(G, B)$ is a $\mathfrak{g}$-topological soft set over $U_{1}$.

Min [14] has established similarity between two soft sets over fixed initial universe $U$ as the above-mentioned. We can give following definition as generalization of similarity relation between soft sets over two different generalized topological spaces using $\mathfrak{g}$-homeomorphism.

DEFINITION 3.19. Let $\left(U_{1}, \mathfrak{g}_{1}\right)$ and $\left(U_{2}, \mathfrak{g}_{2}\right)$ be two generalized topological spaces, $(F, A)$ be a $\mathfrak{g}$-topological soft set over $U_{1}$ with $A \subseteq E_{1}$ and $(G, B)$ be a $\mathfrak{g}$-topological soft set over $U_{2}$ with $B \subseteq E_{2}$ and $\varphi: U_{1} \rightarrow U_{2}$ be a g-homeomorphism. We called that $(F, A)$ is homeomorphically similar to $(G, B)$ if there exists a bijection $\phi: A \rightarrow B$ such that $(\varphi \circ F)(e)=(G \circ \phi)(e)$ for each $e \in A$, and denoted by $(F, A) \approx(G, B)$.

It is easily seen that if we take the identity function $1_{U}:(U, \mathfrak{g}) \rightarrow(U, \mathfrak{g})$, then we have Definition 2.10 .

EXAMPLE 3.20. Let $U_{1}=\{a, b, c\}$ and $U_{2}=\{x, y, z\}$ be initial universes, and $\mathfrak{o}_{1}=\left\{\varnothing, U_{1},\{a\},\{b\},\{c\}\right\}$ and $\mathfrak{o}_{2}=\left\{\varnothing, U_{2},\{x\},\{y\},\{z\}\right\}$ be topologies on $U_{1}$ and $U_{2}$, respectively. Let $\varphi: U_{1} \rightarrow U_{2}$ be an o-homeomorphism such that $\varphi=\{(a, y),(b, x),(c, z)\} . \quad$ And let $(F, A)=\{1=\{a\}, 2=\{c\}\} \quad$ and $(G, B)=\{9=\{y\}, 10=\{x\}\}$ be topological soft sets over $U_{1}$ and $U_{2}$ respectively. So if we define the bijection $\phi: A \rightarrow B$ such that $\phi=\{(1,9),(2,10)\}$, then we obtain;

$$
(\varphi \circ F)(1)=\varphi(F(1))=\varphi(\{a\})=\{y\}=G(9)=G(\phi(1))=(G \circ \phi)(1)
$$

and

$$
(\varphi \circ F)(2)=\varphi(F(2))=\varphi(\{c\})=\{z\}=G(10)=G(\phi(2))=(G \circ \phi)(2) .
$$

Consequently, $(F, A) \approx(G, B)$.

THEOREM 3.21. Let $U_{1}$ and $U_{2}$ be generalized topological spaces.

(a) If any $(F, A) \mathfrak{g}$-homeomorphically similar to the null soft set $\Phi_{B}$ with respect to $B$, then $(F, A)$ is also null.

(b) If any $(F, A) \mathfrak{g}$-homeomorphically similar to the absolute soft set $\mathscr{U}_{B}$ with respect 
to $B$, then $(F, A)$ is also absolute.

Proof. (a) Assume that $\Phi_{B}=(G, B)$. Since $(F, A) \approx \Phi_{B}=(G, B)$, there exists a bijection $\phi: A \rightarrow B$ such that $(\varphi \circ F)(a)=(G \circ \phi)(a)$ for each $a \in A$ where $\varphi$ is a homeomorphism. Since $(G, B)$ is null, we have

$$
\begin{aligned}
(\varphi \circ F)(a) & =(G \circ \phi)(a) \\
(\varphi \circ F)(a) & =\varnothing \\
\varphi(F(a)) & =\varnothing \\
F(a) & =\varnothing
\end{aligned}
$$

for each $a \in A$. Thus $(F, A)$ is null soft set over $U_{1}$.

Proof of (b) is done in a similar way to (a).

In [8], Ge et al. gave some notation for soft sets in a topological space. Their investigations are given as follows;

Let $(U, \mathfrak{o})$ be a topological space, then

(1) $(I, U)$ denotes an identical soft set, where $I(x)=\{x\}$ for each $x \in U$.

(2) $(N, U)$ denotes a neighborhood soft set, where $N(x)=B_{x}$ for each $x \in U$.

(3) $(D, U)$ denotes a derived soft set, where $D(x)=\{x\}^{\prime}$ for each $x \in U$.

(4) $(C, U)$ denotes a closure soft set, where $C(x)=\overline{\{x\}}$ for each $x \in U$.

(5) $\left(D^{c}, U\right)$ denotes a derived-complement soft set, where $D^{c}(x)=\left(\{x\}^{c}\right)^{\prime}$ for each $x \in U$.

(6) $\left(C^{c}, U\right)$ denotes a closure-complement soft set, where $C^{c}(x)=\overline{\left(\{x\}^{c}\right)}$ for each $x \in$ $U$.

(7) $\left(D^{2}, U\right)$ denotes a bi-derived soft set, where $D^{2}(x)=\left(\{x\}^{\prime}\right)^{\prime}$ for each $x \in U$.

(8) $(C D, U)$ denotes a closure-derived soft set, where $C D(x)=\overline{\left(\{x\}^{\prime}\right)}$ for each $x \in U$.

We can obtain some results using above notations.

Note that, the neighborhood soft set $(N, U)$ is obviously topological if $N(x)=B_{x} \in \mathfrak{o}$ for each $x \in U$. 
TheOREM 3.22. Let $(U, \mathfrak{o})$ be a topological space and $(I, U)$ be the identical soft set over $U$. If $(I, U) \mathfrak{o}$-topological soft set over $U$, then $\mathfrak{o}=\mathscr{P}(U)$.

Proof. Obvious.

THEOREM 3.23. Let $(U, \mathfrak{o})$ be a topological space. If $(I, U)$ similar to $(C, U)$, then $\phi$ is identity and $\mathfrak{o}=\mathscr{P}(U)$.

Proof. If $(I, U)$ similar to $(C, U)$, then there exists a bijection $\phi: U \rightarrow U$ such that $(C \circ \phi)(x)=I(x)$ for each $x \in U$. Therefore $C(\phi(x))=\{x\}$ and $\overline{\{\phi(x)\}}=\{x\}$ for each $x \in U$, so $\overline{\{x\}}=\{x\}$. Consequently $\phi$ is identity and $\mathfrak{o}=\mathscr{P}(U)$.

From Definition 3.19 and Ge et al.'s construction for topological spaces in [8], we obtain following theorem.

THEOREM 3.24. Let $\left(U_{1}, \mathfrak{o}_{1}\right)$ and $\left(U_{2}, \mathfrak{o}_{2}\right)$ be topological spaces. If $U_{1}$ homeomorphic to $U_{2}$, then

(1) $\left(I, U_{1}\right) \approx\left(I, U_{2}\right)$,

(2) $\left(N, U_{1}\right) \approx\left(N, U_{2}\right)$,

(3) $\left(D, U_{1}\right) \approx\left(D, U_{2}\right)$,

(4) $\left(C, U_{1}\right) \approx\left(C, U_{2}\right)$,

(5) $\left(D^{c}, U_{1}\right) \approx\left(D^{c}, U_{2}\right)$,

(6) $\left(C^{c}, U_{1}\right) \approx\left(C^{c}, U_{2}\right)$,

(7) $\left(D^{2}, U_{1}\right) \approx\left(D^{2}, U_{2}\right)$,

(8) $\left(C D, U_{1}\right) \approx\left(C D, U_{2}\right)$.

PROOF. We prove only (4). Other implications is proved by similar way. If $U_{1}$ homeomorphic to $U_{2}$, then there exists a homeomorphism $\varphi$ from the topological space $U_{1}$ to the topological space $U_{2}$. Therefore, we have $\varphi(\bar{X})=\overline{\varphi(X)}$ for each $X \subseteq U_{1}$. Hence, we obtain

$$
\left(\varphi^{*} \circ C\right)(x)=\varphi^{*}(C(x))=\varphi^{*}(\overline{\{x\}})=\overline{\{\varphi(x)\}}=C(\varphi(x))=(C \circ \varphi)(x)
$$

for all $x \in U_{1}$, where $\varphi^{*}$ is a function induced by $\varphi$ from $\mathscr{P}\left(U_{1}\right)$ to $\mathscr{P}\left(U_{2}\right)$.

In [11], Li et al. gave a relation between topological spaces and soft sets. They defined the concept of topological soft set, restricted form than our definition, as follows; 
Definition 3.25. [11] Let $(F, A)$ be a soft set over $U$. Then $(F, A)$ is called topological, if $\{F(a) \mid a \in A\}$ is a topology on $U$.

We can give some soft set theoretic results using this definition.

THEOREM 3.26. If $(F, A)$ and $(G, B)$ topological soft set in the sense of Definition 3.25 and $(F, A)$ is similar to $(G, B)$, then the topologies induced by $(F, A)$ and $(G, B)$ are same.

PROOF. It is obvious from Definition 2.10.

In [20], Shabir and Naz gave the concept of soft topology over an initial universe $U$ as follows;

DEFINITION 3.27. [20] Let $\widetilde{\mathfrak{o}}$ be the collection of soft sets over $U$, then $\widetilde{\mathfrak{o}}$ is said to be a soft topology on $U$ if

(1) $\Phi, \widetilde{U}$ belong to $\widetilde{\mathfrak{o}}$

(2) the union of any number of soft sets in $\widetilde{\mathfrak{o}}$ belongs to $\widetilde{\mathfrak{o}}$

(3) the intersection of any two soft sets in $\widetilde{\mathfrak{o}}$ belongs to $\widetilde{\mathfrak{o}}$.

The triplet $(U, \widetilde{\mathfrak{o}}, E)$ is called a soft topological space over $U$. The members of $\widetilde{\mathfrak{o}}$ are said to be soft open sets in $U$.

In [20], Shabir and Naz demonstrated that if $(U, \widetilde{o}, E)$ is a soft topological space over $U$, then the family $\mathfrak{o}_{e}=\{F(e) \mid(F, E) \in \widetilde{\mathfrak{o}}\}$ for each $e \in E$, defines a topology on $U$. Therefore if we have a soft topology $\widetilde{\mathfrak{o}}$ over $U$, then every member of $\widetilde{\mathfrak{o}}$ is a $\mathfrak{o}$ topological soft set over $U$.

Note that, if we have a $\gamma$-topological soft set $(F, A)$ over $U$ then $F(e) \subseteq \gamma(F(e))$ for all $e \in A$ from Definition 3.1. So, this implies that $(F, A) \widetilde{\subset} \gamma(F, A)$. Therefore, we can say that $(F, A)$ is $\gamma$-open soft set in Császár' s sense. Now, let $(U, \mathfrak{g})$ be generalized topological spaces and $E$ be fixed parameter set. Consider the family

$$
\widetilde{\mathfrak{g}}=\{(F, E) \mid(\forall e \in E)(F(e) \in \mathfrak{g})\} .
$$

Then we have following theorem.

THEOREM 3.28. $(U, \widetilde{\mathfrak{g}}, E)$ is a generalized soft topological space.

ProOF. Since $\mathfrak{g}$ is a generalized topology on $U$, then $\varnothing \in \mathfrak{g}$. At that case, for all $e \in E, F(e)=\varnothing \in \mathfrak{g}$. So, we obtain $(F, E)=\widetilde{\Phi} \in \widetilde{\mathfrak{g}}$. Therewithal, consider the subfamily $\left\{\left(F_{i}, E\right) \mid i \in I\right\} \subset \widetilde{\mathfrak{g}}$. For all $i \in I$ and $e \in E, F_{i}(e) \in \mathfrak{g}$. Since $\mathfrak{g}$ is a generalized topology 
on $U$, then for all $e \in E, \bigcup_{i \in I} F_{i}(e) \in \mathfrak{g}$. Thus, we obtain $\widetilde{\cup}_{i \in I}\left(F_{i}, E\right) \in \widetilde{\mathfrak{g}}$. Hence, $\widetilde{\mathfrak{g}}$ is a generalized soft topology on $U$.

Particularly, if we take topological space instead of generalized topological space, we get soft topology on this way i.e.

$$
\widetilde{\mathfrak{o}}=\{(F, E) \mid(\forall e \in E)(F(e) \in \mathfrak{o})\}
$$

is a soft topology on $U$. As a result, we can achieve a soft topology from topological soft sets on any given topological space.

In [16], Molodtsov pointed out that every topological space is considered a soft set. Let $(U, \mathfrak{o})$ be a topological space. Define the mapping $T: U \rightarrow \mathscr{P}(\mathfrak{o})$ such that $\forall u \in U$, $T(u)=\{V \in \mathfrak{o} \mid u \in V\}$ i.e. $T(u)$ is the family of open neighborhoods of $u$. Thus, $(T, U)$ is a soft set over $\mathfrak{o}$.

THEOREM 3.29. Let $\mathfrak{o}$ and $\mathfrak{o}^{\prime}$ be topologies on $U$, and $(T, U)$ and $\left(T^{\prime}, U\right)$ be $\mathfrak{o}$ topological soft sets over $\mathfrak{o}$ and $\mathfrak{o}^{\prime}$ respectively. If $(T, U) \cong\left(T^{\prime}, U\right)$, then $\mathfrak{o}=\mathfrak{o}^{\prime}$.

Proof. Suppose that $A \in \mathfrak{o}$. There is $x \in A \in \mathfrak{o}$. So, $A$ is a open neighborhood of $x$ i.e. $A \in T(x)$. Since $(T, U) \cong\left(T^{\prime}, U\right)$, there exist the bijective function $\phi: U \rightarrow U$ such that $T(x)=\left(T^{\prime} \circ \phi\right)(x)$ for each $x \in X$. Therefore, $A \in T(x)=\left(T^{\prime} \circ \phi\right)(x)$. We obtain that $A$ is a open neighborhood of $\phi(x)$. Thus $A \in \mathfrak{o}^{\prime}$.

Similar to the other inclusion is shown. Thus $\mathfrak{o}=\mathfrak{o}^{\prime}$

COROLlary 3.30. If $(T, U) \cong\left(T^{\prime}, U\right)$, then $\phi: U \rightarrow U$ is an identical homeomorphism.

In [3], P. Alexandroff introduced the Alexandroff space which is a topological space such that the intersection of every family of open sets is open (or equivalently every point has a minimal neighborhood). These spaces are also called finitely generated spaces since their topology is uniquely determined by the family of all finite subspaces. Alexandroff spaces can be viewed as a generalization of finite topological spaces.

With the above expression, we can obtain a soft set over a universe if we have a Alexandroff topology on this universe. At the same time, starting from the above expression over the universe, we can get an o-topological soft set over the universe as follows. We know that if $T: U \rightarrow \mathscr{P}(\mathfrak{o})$ such that $\forall u \in U, T(u)=\{A \in \mathfrak{o} \mid u \in A\}$ then $(T, U)$ is a soft set over $\mathfrak{o}$. If we define the mapping $T^{*}: U \rightarrow \mathscr{P}(U)$ such that $T^{*}(u)=\bigcap\{A \in \mathfrak{o} \mid u \in A\}$, we obtain a soft set over $U$, and it is an o-topological soft 
set since the intersection of open neighborhoods is open in Alexandroff spaces. Thus for all soft sets $(T, U)$ over a Alexandroff space $U$, we have an o-topological soft set over $U$.

EXAMPLE 3.31. Let $U=\{a, b, c\}, \mathfrak{o}=\{\varnothing, U,\{a\},\{a, b\}\}$ be a topology over $U$. Since $U$ is finite, then $\mathfrak{o}$ is an Alexandroff space. For the mapping $T: U \rightarrow \mathscr{P}(\mathfrak{o})$, let

$$
(T, U)=\{a=\{\{a\},\{a, b\}, U\}, b=\{\{a, b\}, U\}, c=\{U\}\} .
$$

$T^{*}$ is induced by $T$ as following.

$$
\begin{gathered}
T^{*}(a)=\bigcap\{\{a\},\{a, b\}, U\}=\{a\}, \\
T^{*}(b)=\bigcap\{\{a, b\}, U\}=\{a, b\}
\end{gathered}
$$

and

$$
T^{*}(c)=\bigcap\{U\}=U .
$$

Thus we obtain the soft set $\left(T^{*}, U\right)=\{a=\{a\}, b=\{a, b\}, c=U\}$ over $U$.

So, we obtain the following theorem for Alexandroff spaces.

THEOREM 3.32. Let $(U, \mathfrak{o})$ and $\left(V, \mathfrak{o}^{\prime}\right)$ be Alexandroff topological spaces. $(U, \mathfrak{o})$ is homeomorphic to $\left(V, \mathfrak{o}^{\prime}\right)$ if and only if $\left(T_{U}^{*}, U\right) \approx\left(T_{V}^{*}, V\right)$.

Proof. Suppose that the space $U$ is homeomorphic to the space $V$. Then there exist a homeomorphism $\phi: U \rightarrow V$. We must show that

$$
\left(\phi \circ T_{U}^{*}\right)(u)=\left(T_{V}^{*} \circ \phi\right)(u)
$$

for all $u \in U$. Since $\phi$ is a homeomorphism, $\phi$ is bijective, continuous and open function. In that case, for all $u \in U$,

$$
\begin{aligned}
\left(\phi \circ T_{U}^{*}\right)(u) & =\phi\left(T_{U}^{*}(u)\right) \\
& =\phi(\bigcap\{A \in \mathfrak{o} \mid u \in A\}) \\
& =\bigcap\left\{\phi(A) \in \mathfrak{o}^{\prime} \mid \phi(u) \in \phi(A)\right\} \\
& =\bigcap\left\{B \in \mathfrak{o}^{\prime} \mid \phi(u) \in B\right\} \\
& =T_{V}^{*}(\phi(u))=\left(T_{V}^{*} \circ \phi\right)(u)
\end{aligned}
$$

Thus we achieve that $\left(T^{*}, U\right) \approx\left(T^{*}, V\right)$.

On the other hand, suppose that $\left(T^{*}, U\right) \approx\left(T^{*}, V\right)$. Then we have a bijective function 
$\phi: U \rightarrow V$ such that $\left(\phi^{*} \circ T^{*}\right)(u)=\left(T^{*} \circ \phi\right)(u)^{1}$ for all $u \in U$. We need to show that $\phi$ is a homeomorphism from $U$ to $V$. We know that $\phi$ is bijection. Since for all $u \in U$, $T^{*}(u)=\bigcap\{A \in \mathfrak{o} \mid u \in A\}$ is an open set in $U$ and $\left(\phi^{*} \circ T^{*}\right)(u)=\left(T^{*} \circ \phi\right)(u)$, we obtain that $\phi$ is an open function. Afterwards, since $\phi$ is a bijection, there exist its inverse $\phi^{-1}: V \rightarrow U$. Then we have $\left(\phi^{-1}\left(T^{*}(v)\right)=T^{*}\left(\phi^{-1}(v)\right)\right.$ for all $v \in V$. Since $T^{*}(v)$ is an open set for all $v \in V$ in the space $V, \phi^{-1}$ is an open function i.e. $\phi$ is continuous. Consequently, $\phi$ is a homeomorphism from $U$ to $V$.

Of course, the above theorem is not valid for arbitrary topological spaces which is defined by $\mathfrak{o}$-topological soft set in the above manner, since the intersection of arbitrary number of open sets may not be open. However, the intersection of every closed sets is closed in arbitrary topological space. We can define the soft set using closed sets in given any topological space. Let' s consider a topological space $(U, \mathfrak{o})$. Inspired from Molodtsov, define the mapping $K: U \rightarrow \mathscr{P}(\mathfrak{c})$ such that $\forall u \in U, K(u)=\{A \in \mathfrak{c} \mid u \in A\}$ i.e. $K(u)$ is the family of closed neighborhoods of $u$. Thus, $(K, U)$ is a soft set over c. If we define the mapping $K^{*}: U \rightarrow \mathscr{P}(U)$ such that $K^{*}(u)=\bigcap\{A \in \mathfrak{c} \mid u \in A\}$ which is induced by $K$. So, $\left(K^{*}, U\right)$ is a c-topological soft set over $U$.

We can obtain following theorem for arbitrary topological spaces.

THEOREM 3.33. Let $(U, \mathfrak{o})$ and $\left(V, \mathfrak{o}^{\prime}\right)$ be topological spaces. Then $(U, \mathfrak{o})$ is homeomorphic to $\left(V, \mathfrak{o}^{\prime}\right)$ if and only if $\left(K_{U}^{*}, U\right) \approx\left(K_{V}^{*}, V\right)$ for each $u \in U$.

PROOF. Similar to proof of Theorem 3.32.

COROLlary 3.34. Let $(U, \mathfrak{g})$ and $\left(V, \mathfrak{g}^{\prime}\right)$ be generalized topological spaces. $(U, \mathfrak{g})$ is generalized homeomorphic to $\left(V, \mathfrak{g}^{\prime}\right)$ if and only if $\left(K_{U}^{*}, U\right) \approx\left(K_{V}^{*}, V\right)$ where $K^{*}(u)=\bigcap\{A \in \mathfrak{g c} \mid u \in A\}$.

\section{CONCLUSION}

In this paper, we have introduced the concept of topological soft set without any restrictions, and we have examined the relationship between them. We have been achieved some results given the notations by Ge et al [8]. We have pointed out that each element of soft topology given by Shabir and Naz in [20] is a topological soft set over a relevant universe and conversely we have pointed out that soft topology and generalized soft topology is obtained when we have topological soft sets. Finally,

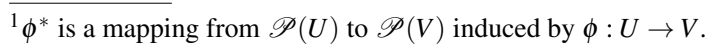


we have obtained topological soft set for a topological space using Molodtsov's deduction in [16], and we have proved that two space is homeomorphic if and only if topological soft sets derived from them are similar.

This study can be a useful source for researchers working in this area. For the future work, we can examine structures of topological soft sets using topological properties such as separation axioms, connectedness, compactness etc.

\section{REFERENCES}

Abd El-Monsef M. E., El-Deeb S. N., Mahmoud R. A., $\beta$-open sets and $\beta$-continuous mappings, Bull. Fac.

Sci. Assiut Univ. 12 (1983), 77-90.

Aktaş H., Çağman N., Soft sets and soft groups, Inform. Sciences. 177 (2007) 2726-2735.

Alexandroff P., Diskrete Räume, Rec. Math. [Mat. Sbornik] N.S. Vol. 2 (44) (1937), 501-519.

Ali M. I., Feng F., Liu X. Y., Min W. K., Shabir M., On Some New Operations in Soft Set Theory, Comput. Math. Appl. 57 (2009) 1547-1553.

Császár Á., Generalized open sets, Acta Math. Hungar. 75 (1997), 65-87.

Császár Á., Generalized topology, generalized continuity, Acta Math. Hungar. 96 (4) (2002), 351-357.

Császár Á., Generalized open sets in generalized topology, Acta Math. Hungar. 106 (2005), 53-66.

Ge X., Li Z., Ge Y., Topological spaces and soft sets, J. Comput. Anal. Appl. 13 (4) (2011) 881-885.

Kharal A., Ahmad B., Mappings on soft classes, New Math. and Nat. Computation 7 (3) (2011) 471-481.

Levine N., Semi-open sets and semi-continuity in topological spaces, Amer. Math. Monthly 70 (1963), 36-41.

Li Z., Chen H., Gao N., The topological structure on soft sets, J. Comput. Anal. Appl. 15 (4) (2013) 746752.

Maji P. K., Biswas R., Roy A. R., Soft set theory, Comput. Math. Appl. 45 (2003) 555-562.

Mashhour A. S., Abd El-Monsef M. E., El-Deeb S. N., On precontinuous and weak precontinuous mappings, Proc. Math. Phys. Soc. Egypt 53 (1982), 47-53.

Min W. K., Similarity in soft set theory, Appl. Math. Lett. 25 (2012) 310-314.

Min W. K., Soft sets over a common topological universe, Journal of Intelligent \& Fuzzy Systems, vol. 26 (5) (2014) 2099-2106.

Molodtsov D., Soft sets-first results, Comput. Math. Appl. 37 (1999) 19-31.

Njåstad O., On some classes of nearly open sets, Pasific J. Math. 15 (1965), 961-970.

Pei D., Miao D., From Soft Sets to Information Systems, Proceedings of IEEE International Conference on Granular Computing 2 (2005) 617-621.

Qin K., Hong Z., On soft equality, J. Comput. Appl. Math. 234 (2010) 1347-1355.

Shabir M., Naz M., On soft topological spaces, Comput. Math. Appl. 61 (2011) 1786-1799. 


\author{
Mustafa Burç Kandemir \\ Department of Mathematics, Faculty of Science, \\ Mugla Sıtkı Koçman University, \\ 48000, Mugla, Turkey. \\ email: mbkandemir@mu.edu.tr \\ Bekir Tanay \\ Department of Mathematics, Faculty of Science, \\ Mugla Sıtkı Koçman University, \\ 48000, Mugla, Turkey. \\ email: btanay@mu.edu.tr
}

